\title{
Random access improvement for M2M communication in LTE-A using femtocell
}

\author{
Eric Sackey, Rajeev Paulus \\ Department of E.C.E., Sam Higgingbottom University of Agriculture, Technology and Sciences, India
}

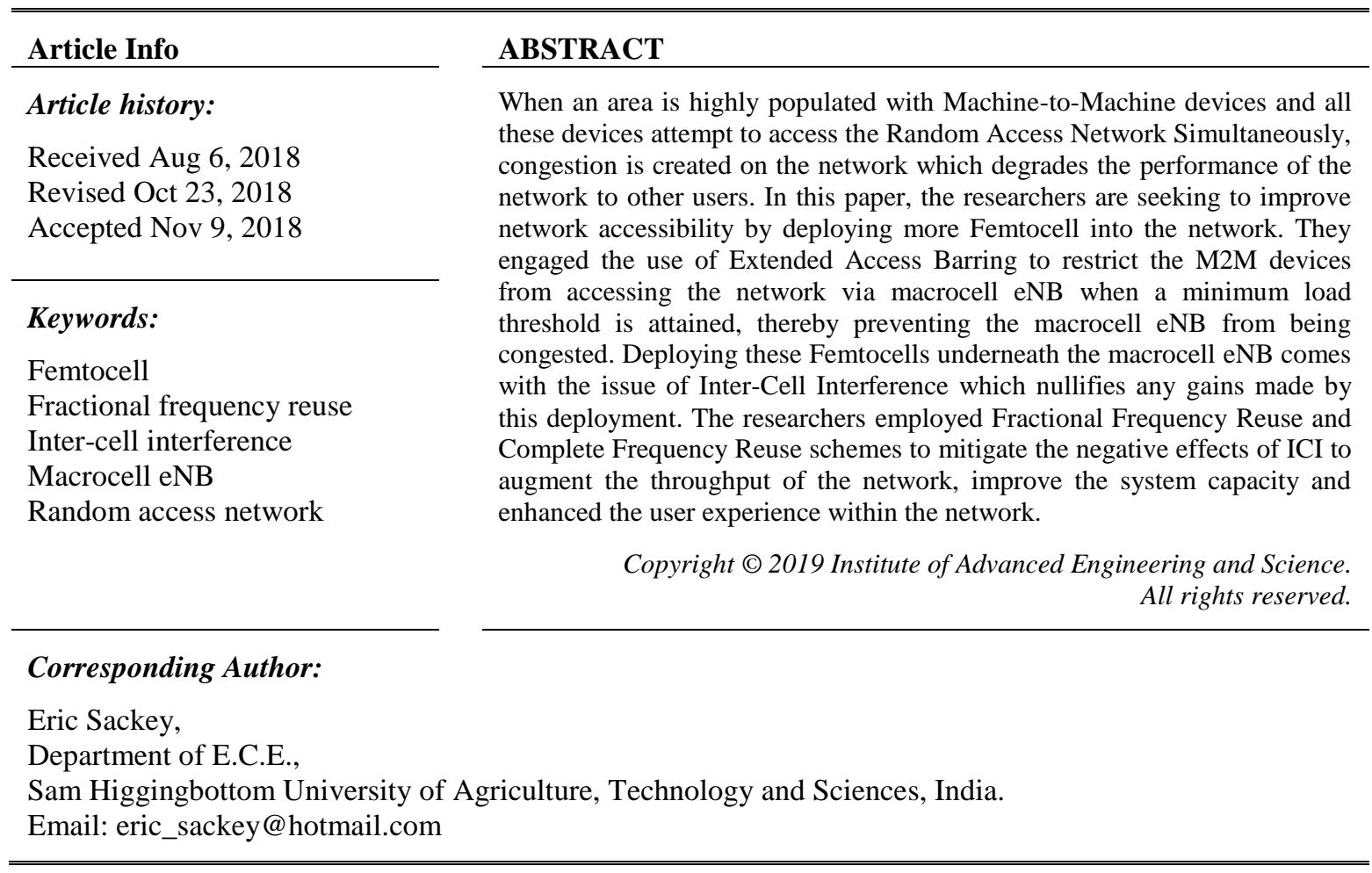

\section{INTRODUCTION}

The increasing demand for high data access network coupled with ubiquitous connectivity necessitated the commissioning of Long Term Evolution (LTE) which evolved into Long Term Evolution Advanced (LTE-A). The LTE primary objective is to improve system capacity, coverage area, low latency, reduce operational cost, flexible bandwidth operation and seamless integration of the existing systems [1]. The LTE-A on the other hand mainly targets improving the efficiency of the spectrum per unit area of the existing systems. This can be accomplished by supporting the deployment of low power rated Heterogeneous Network (HetNet) comprising of Microcell, Picocell, Femtocell and Relay Nodes underneath the Macrocell eNB lay out to improve the spectral efficiency per unit area and to enhance the user experience within the network [2], [3]. With the envisioned smart cities projects it is expected that more machine to machine (M2M) devices will be introduced into the existing cellular network [4]. It is projected that by the end of 2020 there will over 50 billion connected devices [5], [6]. When an area is highly populated by M2M devices and all these devices try to access the network simultaneously congestion is created on the network, which in turn degrades the performance of the network. One way to mitigate this problem is by injecting more access points into the network, and this can be done by deploying HetNet underneath the macrocell eNB [7]. The M2M communication devices are expected to be the focal point around which the future Internet of Things (IoT) is expected to be built.

Both LTE and LTE-A are built on Orthogonal Frequency Division Multiple Access (OFDMA) technology. In OFDMA, transmission and multiple access techniques are combined in the downlink (DL). The bandwidths in OFDMA systems are splitted into multiple sub-carriers for parallel transmission of data. These sub-carriers are mutually orthogonal to mitigate the effect of inter-carrier interference (ICI). 
By employing suitable cyclic prefixes, ICI and inter symbol interference (ISI) can be completely eliminated [8]. The major challenge in LTE/LTE-A is the issue of inter-cell interference or co-channel interference $(\mathrm{CCI})$ which limits the performance of the network especially for users along the cell edges zones [9]. Fractional Frequency Reuse (FFR) has recently emerged as an appropriate technique to combat the effect of inter-cell interference in LTE/LTE-A based HetNet [10], [11].

This paper considers, incorporating femtocell base station underneath the macrocell evolved Node Base station (eNB) to improve the capacity of the cell and to resolve the Random Access congestion by allowing the M2M devices to access the network via the femtocell by employing Complete Frequency Reuse (CFR) management scheme. Since the macrocell eNB has a much stronger signal than the femtocell, most of the M2M devices will want to access the network through the macrocell eNB other than the femtocell. Adaptive access class barring mechanism will be employed in the macrocell eNB, once a certain minimum threshold value is attained, class access barring is then activated to prevent any further use of the macrocell eNB by the M2M devices [12], [13].

The rest of the paper is organised as follows: Section 1.A gives an overview of some related works. Section 2 discusses the resource allocation scheme to be employed in this work. Section 3 presents a mathematical analysis of the system capacity, success probability of access gain and the probability of collision as the number M2M device increases. Section 4 presents the analysis of the simulation output results and finally section 5 concludes the paper.

In [14] interference avoidance scheme was proposed in WiMax networks comprising of femtocells. Frequency was allocated dynamically taking into account the users bandwidth requirement. The objective was to reduce interference, thereby improve network system capacity. The drawback was that the femtocells were operated and managed by individual owners. In [15] various Frequency resuse schemes in LTE networks were examined to overcome intercell interference. Fractional Frequency Reuse and Two Level Power Control (TLPC) schemes were employed to ensure suitable inner ratio and power ratio were providered. Best performance was attained when fair throughput was assumed and intercell interference was reduced considerably. In [16] the impart of FFR in WiMax networks was studied and the result was that when FFR with a reuse factor 3 was implemented, it reduced intercell interference at the cell edge zone better with a corresponding increase in throughput as compared those with reuse factor 1. In [17] the performance of FFR was analysed in terms of user allocation. Three method of zones allocation were considered, SINRbase, Distance-base and load-balance method. New methods emerged when SINR was combined with loadbalance and SINR combined with user assigned per load available. Combing these two gave better performance then when SINR or distance was considered individually.

\section{RESOURCE ALLOCATION}

This section compares the two resource allocation schemes to be used in assigning the available resources between the macrocell eNB and the femtocells. The Fractional frequency Reuse scheme divides the coverage area of a cell into two; the inner zone and outer zone. In the Complete Frequency Reuse Scheme the entire available bandwidth is shared among the neighbouring macrocell eNBs and the femtocells are distributed among the eNBs in such a manner that a femtocell in a particular cell can use the bandwidth of all the neighbouring cell exception of the cell in which it is located.

\subsection{Fractional frequency reuse (FFR)}

The FFR scheme was developed to overcome the limitation of the conventional frequency reuse scheme. In the FFR scheme, the eNB coverage area is divided into inner and outer zones where a portion of the available bandwidth is assigned to the inner zone with a reuse factor of one $(R F=1)$ and the remaining bandwidth assigned to the outer zone with a higher reuse factor say three or higher. The cells in the outer zone are arranged in such a manner so as to eliminate the effect of such inter-cell interference from neighbouring cells [18] as shown in the Figure 1. This scheme permits the allocation of identical power level in the downlink at high data rate [19]. One very important characteristic of this scheme as pointed out in [20] is that the radius of the inner zone macrocell eNB must be at most 0.65 times the radius of the outer zone macrocell eNB and must be uniformly distributed to ensure throughput maximization [21]. 

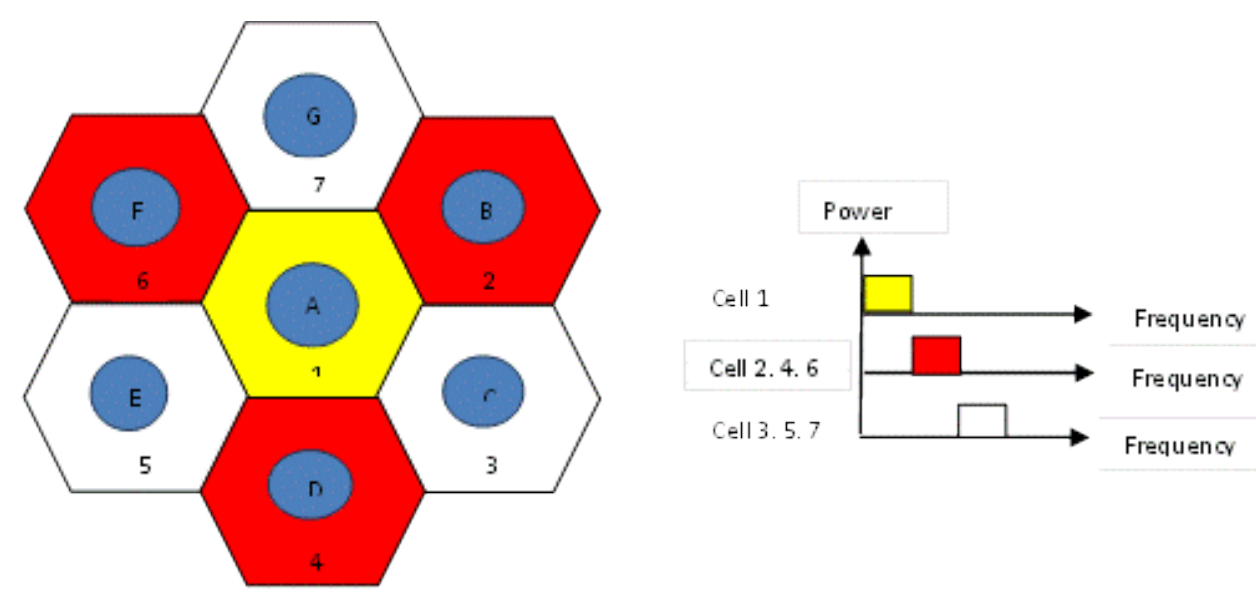

Figure 1. Fractional frequency reuse scheme

\subsection{Complete frequency reuse (CFR) scheme}

In this scheme, the total available bandwidth is share among the neighbouring eNB cells with a reuse factor of say seven $(\mathrm{RF}=7)$. The Femtocell are distributed among these eNB in such a manner that a femtocell in any particular eNB cell can use the bandwidth of all surrounding eNB cell except the eNB cell in which it is located. The CFR scheme is superior to the FFR in the sense that it overcomes the limitation of FFR. In the CFR, the frequency band in the outer zone can be partitioned into any number of sub-bands, this way interference with neighbouring cells is avoided. This provides flexibility in the sub-band allocation in the outer zone which is very important in the real-life network condition. Complete Frequency Reuse Scheme shown in Figure 2.

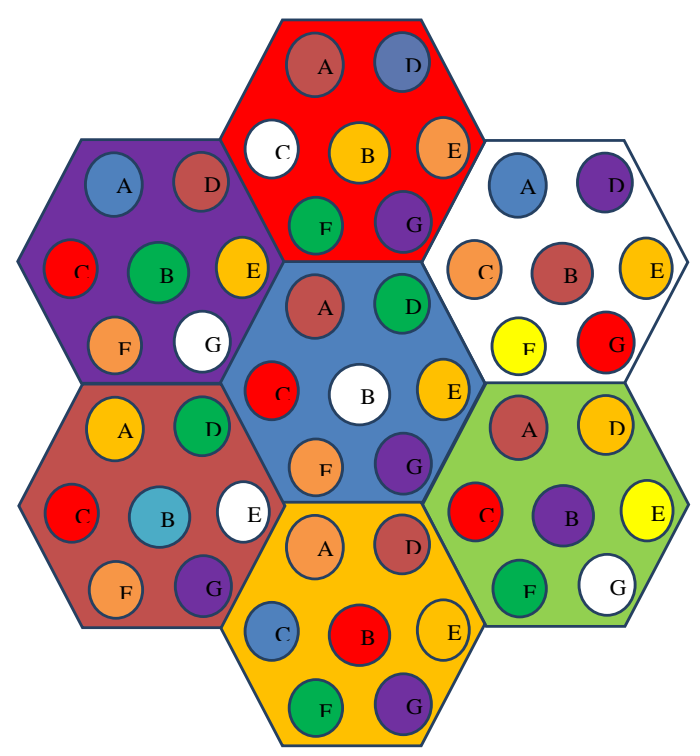

Figure 2. Complete frequency reuse scheme

\section{PROBLEM FORMULATION}

This section presents a mathematical model of the system in terms output probabilities.

\subsection{System Capacity}

The capacity for M2M on LTE-A system may be defined as the maximum number of successful request granted in accessing "M" resources in a single slot [22]. There are 64 possible resources available in each slot [23]. The capacity of eNB can be calculated as adopted from [24]. 


$$
C=\left[\log \left(\frac{M}{M-1}\right)\right]^{-1} \times\left(1-\frac{1}{M}\right)^{\left[\log \left(\left(\frac{M}{M-1}\right)\right)\right]^{-1}-1}
$$

where $\mathrm{M}$ represents the number of resources available to the eNB. Likewise the capacity of the Femtocell can be presented as

$$
C_{F C}=\left[\log \left(\frac{M_{F C}}{M_{F C}-1}\right)\right]^{-1} \times\left(1-\frac{1}{M_{F C}}\right)^{\left[\log \left(\frac{M_{F C}}{M_{F C^{-1}}}\right)\right]^{-1}-1}
$$

where $\mathrm{M}_{\mathrm{FC}}$ represents the resources assigned to the femtocell. In the FFR scheme since the total available bandwidth is shared between the eNB in the inner zone and the femtocell in the outer zone as depicted in the Figure 1 of the FFR scheme. The total resource is given by

$$
M=M_{A}+M_{1}+M_{2}+M_{3}
$$

The capacity of the FFR scheme is therefore given as

$$
C_{F F R}=\left[\log \left(\frac{M}{M-1}\right)\right]^{-1} \times\left(1-\frac{1}{M}\right)^{\left[\log \left(\frac{M}{M-1}\right)\right]^{-1}-1}
$$

$\mathrm{C}_{\mathrm{FFR}}=\mathrm{C}$ as in Equation (1). If there are $\beta$ numbers of eNB in the FFR scheme, then the capacity can be obtained by

$$
C_{F F R}=C \times \beta
$$

In the CFR scheme however, the femtocell in any particular eNB uses all the available bandwidth in the neighbouring cells except the very eNB cell in which it is located. The CFR scheme therefore has the capability to improve the system's capacity. The capacity of the femtocell is given in (2) as

$$
C_{F C}=\left[\log \left(\frac{M_{F C}}{M_{F C}-1}\right)\right]^{-1} \times\left(1-\frac{1}{M_{F C}}\right)^{\left[\log \left(\frac{M_{F C}}{M_{F C}-1}\right)\right]^{-1}-1}
$$

If there are $\mathrm{N}$ Number of femtocells in each eNB cell and if the femtocell have the same resources as the eNB. Then the capacity of CFR scheme is obtained by

$$
C_{C F R}=\left(C_{F C} \times N\right)+C
$$

But the capacity of the femtocell is the same as the capacity of the eNB

$$
\begin{aligned}
& C_{F C}=C \\
& C_{C F R}=C N+C \\
& C_{C F R}=(N+1) \times C
\end{aligned}
$$

If there are $\beta$ eNB cells, then the capacity of the CFR is obtained by

$$
C_{C F R}=(N+1) \times C \beta
$$

The capacity of the CFR scheme has outperformed the capacity of FFR and the legacy LTE system.

\subsection{Probability of success in random access channel gain}

When an area is highly populated with M2M devices, congestion is created on the RACH which in turn degrades the performance of the network when all the devices attempt to access the network simultaneously. This harmful effect can be mitigated by injecting more access (Femtocells) points into the network and allowing the M2M devices access to the network through these Femtocells. In the LTE-A cellular network, probability of a device gaining access to the RACH is given by 


$$
P_{L T E-A}=\left(1-\frac{1}{M}\right)^{V-1}
$$

where $\mathrm{M}$ and $\mathrm{V}$ represent the number of available resource assigned to the eNB and the number of users respectively. In the Femtocell base station, the probability of a device gaining access to the RACH is given by

$$
P_{F C}=\left(1-\frac{1}{M_{F}}\right)^{V_{F}-1}
$$

where $\mathrm{M}_{\mathrm{F}}$ and $\mathrm{V}_{\mathrm{F}}$ represent the resources assigned and number of users on the femtocell respectively. Now if there are $\mathrm{N}$ femtocells in each eNB, then the probability of a M2M device successfully gaining access to the $\mathrm{RACH}$ is obtained by

$$
P_{F S}=\frac{1}{N+1} \times\left(\sum_{n=1}^{N}\left(1-\frac{1}{M_{F}}\right)^{V_{F}-1}+\left(1-\frac{1}{M}\right)^{V-1}\right)
$$

To demonstrate that M2M devices have more success probability when deployed in the Femtocell than when they are deployed in the eNB without degrading the performance of the network.

$$
\begin{aligned}
& \mathrm{P}_{\mathrm{FS}}-\mathrm{P}_{\mathrm{LTE}-\mathrm{A}}>0 \\
& \Rightarrow \frac{1}{N+1} \times\left(\sum_{n=1}^{N}\left(1-\frac{1}{M_{F}}\right)^{V_{F}-1}+\left(1-\frac{1}{M}\right)^{V-1}\right)-\left(1-\frac{1}{M}\right)^{V-1}>0 \\
& \Rightarrow\left\{\frac{1}{N+1} \times\left(\sum_{n=1}^{N}\left(1-\frac{1}{M_{F}}\right)^{V_{F, n}-1}+\left(1-\frac{1}{M}\right)^{V-1}\right)\right\}-\left(1-\frac{1}{M}\right)^{V-1}>0 \\
& \Rightarrow \frac{1}{N+1} \times\left\{\sum_{n=1}^{N}\left(1-\frac{1}{M_{F}}\right)^{V_{F, n}-1}+\left(1-\frac{1}{M}\right)^{V-1}-N \times\left(1-\frac{1}{M}\right)^{V-1}-\left(1-\frac{1}{M}\right)^{V-1}\right\}> \\
& \Rightarrow \frac{N}{N+1} \times\left\{\left(1-\frac{1}{M_{F}}\right)^{V_{F, n}-1}-\left(1-\frac{1}{M}\right)^{V-1}\right\}>0
\end{aligned}
$$

but $\frac{N}{N+1}>0$

$$
\begin{aligned}
& \Rightarrow\left(1-\frac{1}{M_{F}}\right)^{V_{F}-1}-\left(1-\frac{1}{M}\right)^{V-1}>0 \\
& \Rightarrow \frac{\left(1-\frac{1}{M_{F}}\right)^{V_{F}-1}}{\left(1-\frac{1}{M}\right)^{V-1}}>0
\end{aligned}
$$

List of four cases to drive home this point:

\section{a. Case 1}

The resources assigned to the Femtocell are less than those assigned to the eNB, but the number of users on the femtocell is equal to the number of users on the eNB. Thus $\mathrm{M}_{\mathrm{F}}<\mathrm{M}, \mathrm{V}_{\mathrm{F}}=\mathrm{V}$, by inputting these into (18).

$$
\frac{\left(1-\frac{1}{M_{F}}\right)^{V_{F}-1}}{\left(1-\frac{1}{M}\right)^{V-1}}>0
$$

In which case

$$
\left\{\frac{\left(1-\frac{1}{M_{F}}\right)}{\left(1-\frac{1}{M}\right)}\right\}^{V-1}>0
$$

$P_{F S}<P_{L T E-A}$ 
b. Case 2

The resources assigned to the femtocell are equal to those assigned to the eNB and the number of users on the femtocell is equal to the number of users on the eNB. Thus $M_{F}=M$ and $V_{F}=V$, by inputting these into (18).

$$
\begin{gathered}
\frac{\left(1-\frac{1}{M_{F}}\right)^{V_{F}-1}}{\left(1-\frac{1}{M}\right)^{V-1}}=1 \\
P_{F S}=P_{L T E-A}
\end{gathered}
$$

c. Case 3

The resources assigned to the femtocell are equal to those assigned to the eNB, but the number of user on the femtocell is less than the number of users on the eNB. Thus $M_{F}=M$ but $V_{F}<$ Vutting these into (18).

$$
\frac{\left(1-\frac{1}{M_{F}}\right)^{V_{F}-1}}{\left(1-\frac{1}{M}\right)^{V-1}}>1
$$

Since $\mathrm{M}_{\mathrm{F}}=\mathrm{M}$

$$
\begin{aligned}
& \left(1-\frac{1}{M}\right)^{v_{F}-v}>1 \\
& P_{F S}>P_{L T E-A}
\end{aligned}
$$

This represents the ideal situation since the femtocells have a smaller coverage area and therefore the number of users is less than those on the eNBs, even though they may have the same resources assigned to them as those assigned to the eNBs.

\section{d. Case 4}

The resources assigned to the femtocell are less than those assigned to the eNB and the number of users on the femtocell is also less than the number of users on the eNB. Thus $M_{F}<M$ and $V_{F}<V$.

Here

$$
X=\frac{\left(1-\frac{1}{M_{F}}\right)^{V_{F}-1}}{\left(1-\frac{1}{M}\right)^{V-1}}
$$

where

$$
\log [X]=\left(V_{F}-1\right) \log \left(1-\frac{1}{M_{F}}\right)-(V-1) \log \left(1-\frac{1}{M}\right)
$$

since $\mathrm{M}_{\mathrm{F}}<\mathrm{M}$, it can be concluded that

$$
\begin{aligned}
& \Rightarrow\left(1-\frac{1}{M_{F}}\right)<\left(1-\frac{1}{M}\right) \\
& \Rightarrow \log \left(1-\frac{1}{M_{F}}\right)<\log \left(1-\frac{1}{M}\right) \\
& \Rightarrow\left(V_{F}-1\right) \log \left(1-\frac{1}{M_{F}}\right)<(V-1) \log \left(1-\frac{1}{M}\right) \\
& \log (X)<0 \\
& X \leq 1 \\
& \mathrm{P}_{\mathrm{FS}}<\mathrm{P}_{\mathrm{LTE}-\mathrm{A}}
\end{aligned}
$$

\subsection{Collision probability}

Probability that a device will experience collision on the RACH with other device is expressed as

$$
P_{F C}=1-\left(1-\frac{1}{M_{F}}\right)^{V_{F}-1}
$$


Considering that $\mathrm{N}$ number of femtocell are deployed in each eNB, the probability collision is expressed as

$$
P_{C L T E-A}=\frac{1}{N+1} \times\left\{N+1-\sum_{n=1}^{N}\left(1-\frac{1}{M_{F}}\right)^{V_{F}-1}+\left(1-\frac{1}{M}\right)^{V-1}\right\}
$$

To demonstrate that with the injection of Femtocell, the probability of collision is less than that of the LTE-A system

$$
\begin{aligned}
& P_{C L T E-A}-P_{F C}<0 \\
& \Rightarrow\left\{\frac{1}{N+1} \times\left(N+1-\sum_{n=1}^{N}\left(1-\frac{1}{M_{F}}\right)^{V_{F}-1}+\left(1-\frac{1}{M}\right)^{V-1}\right)\right\}-\left\{1-\left(1-\frac{1}{M}\right)^{V-1}\right\}<0 \\
& \Rightarrow \frac{1}{N+1} \times\left\{N+1-\sum_{n=1}^{N}\left(1-\frac{1}{M_{F}}\right)^{V_{F}-1}+\left(1-\frac{1}{M}\right)^{V-1}-N-1+N \times\left(1-\frac{1}{M}\right)^{V-1}+(1-)^{V-1}\right\} 0
\end{aligned}
$$

The above expression can be simplified as

$$
\begin{aligned}
& \Rightarrow \frac{1}{N+1} \times\left\{2 \times\left(1-\frac{1}{M}\right)^{V-1}+N \times\left(1-\frac{1}{M}\right)^{V-1}-\sum_{n=1}^{N}\left(1-\frac{1}{M_{F}}\right)^{V_{F}-1}\right\}<0 \\
& \Rightarrow \frac{1}{N+1} \times\left\{2 \times\left(1-\frac{1}{M}\right)^{V-1}+N \times\left(1-\frac{1}{M}\right)^{V-1}-\left(1-\frac{1}{M_{F}}\right)^{V_{F}-1}\right\}<0
\end{aligned}
$$

Considering that $\mathrm{N}>0$ and $2 \times\left(1-\frac{1}{M}\right)>0$

$$
\begin{aligned}
& \Rightarrow\left\{\left(1-\frac{1}{M}\right)^{V-1}-\left(1-\frac{1}{M_{F}}\right)^{V_{F}-1}\right\}<0 \\
& \Rightarrow \frac{\left(1-\frac{1}{M}\right)^{V-1}}{\left(1-\frac{1}{M_{F}}\right)^{V_{F}-1}}<0
\end{aligned}
$$

\section{PERFORMANCE ANALYSIS}

Figure 3 gives a comparison between the CFR and FFR schemes and how the number of users can successfully gain access to the Random Access Network (RAN) improves the accessiblity and thereby increasing the capacity of the network. It can be observed from the first plot of Figure 3 that the capacity (in terms of number of users) for the Fractional Frequency Reuse Scheme to the number of eNBs was linear. However that Complete Frequency Reuse is an improvement on that of Fractional Frequency Reuse by a factor of $(\mathrm{N}+1)$. Where $\mathrm{N}$ is the number of femtocells underneath each eNB. This result is an improvement on the use of sector antennas and/or fixed frequency assignment in that when the available bandwidth is divided amongst the various sector antennas this limits the systems capacity. Again with the fixed frequency assignment when a bandwidth is to any particular sector and it is not utilized it goes waste. This technique therefore offers an effective utilization of the available bandwidth especially to the cell egde zone users and the results obtained are fairly similar to those FFR with power level control except that its implementation is simpler.This technique also eliminates the computational complexities associated with dynamic RAN methods. From the onset of this technique the M2M devices can access the network through both the eNBs and the femtocells, once a certain minimum threshold number is attained on the eNBs the access barring mechanism is actuated to prevent the eNBs from being congested and thereby degrading the performance of the eNBs. Meanwhile the M2M devices can continue to access the network through the Femtocells. This implies that the accessibility and capacity of the M2M communication systems can be improved by injecting more femtocells into the network.

Figure 4 depicts the successful probability of a device gaining access to the RAN via femtocells. When an area is highly populated with M2M devices, the possibility of a device gaining access to the RACH becomes very small. With the injection of more HetNet (femtocells) into the network, the possibility of a devices gaining access to the network increases. Since the femtocells have smaller coverage area as compared to the eNBs. It is expected that the number of users on the femtocell must also be small, even though the number of resources assigned to the femtocell are the same as those assigned to the eNBs. 


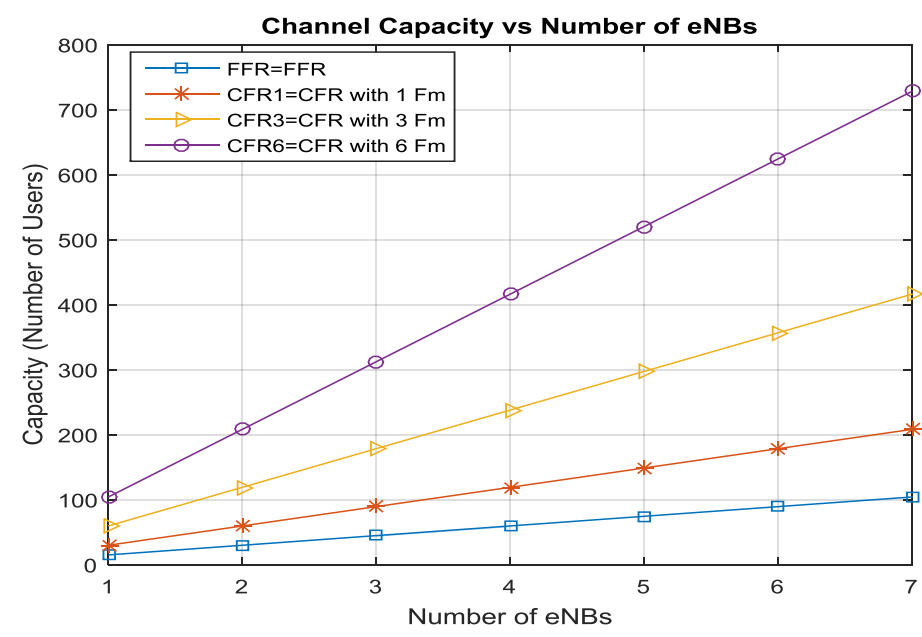

Figure 3. Comparison of channel capacity gain between FFR and CFR

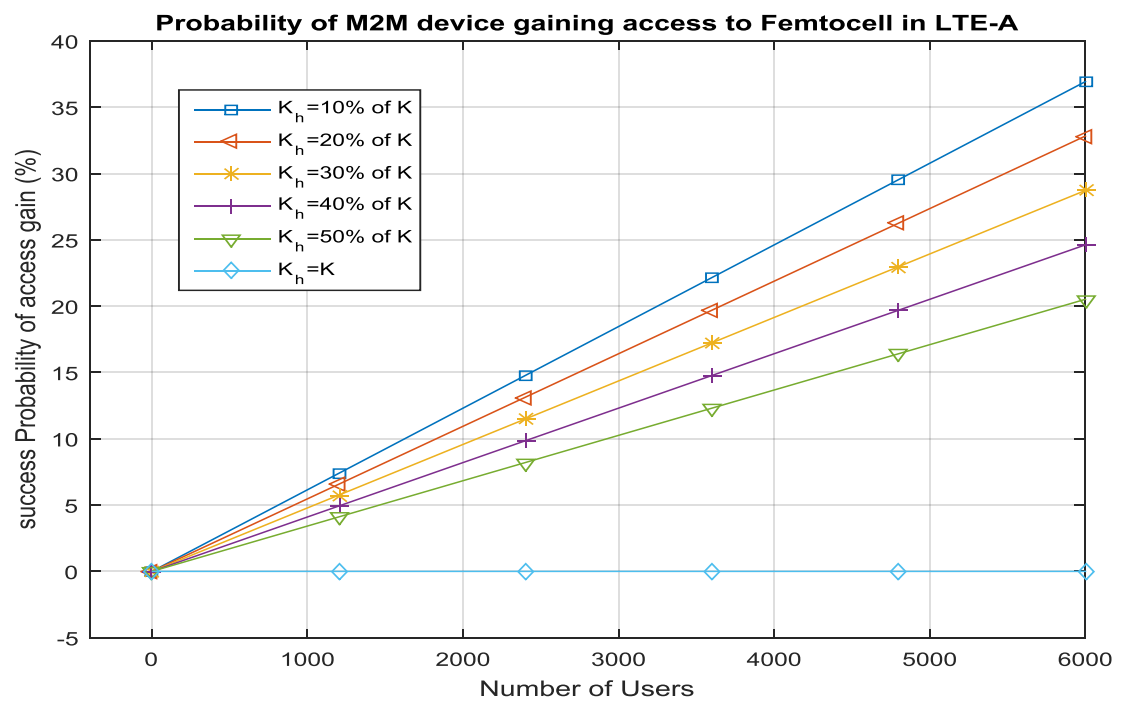

Figure 4. Probability of successful RACH access gain via Femtocell

The number of users on the femtocells is expressed as a percentage of the number of users on the eNBs. It can be inferred from the plots of Figure 4, that when the number of users on the femtocell is low the possibility of a device gaining access to the network was high. As the number of users on the femtocell increases the possibility of a user device gaining access to the network decreases. This trend continued until such a point that the number of users on the femtocell becomes equal to that of the eNB (ie $K_{h}=K$ ) when the success gain is completely lost in which case the performance of the Femtocells become equal to the eNB.

Figure 5 depicts the probability of collision for M2M devices accessing the network. In the first two instances, the M2M devices accessed the network solely through the macrocell eNB with a network capacity of $90 \%$ and $80 \%$ respectively. The third instance depicts the probability of collision when M2M devices accessing the network are evening distributed among the macrocell and two femtocells. The fourth instance depicts the probability of collision when M2M devices accessing the network are evening distributed among the macrocell eNB and three femtocells. From the aforementioned results, it can be observed that for M2M devices accessing the network solely through the macrocell eNB, the probability of collision was very high. However, when the femtocells were injected into the network and the M2M devices were allowed to access the network through both macrocell eNB and the femtocells the probability of collision was significantly reduced. 


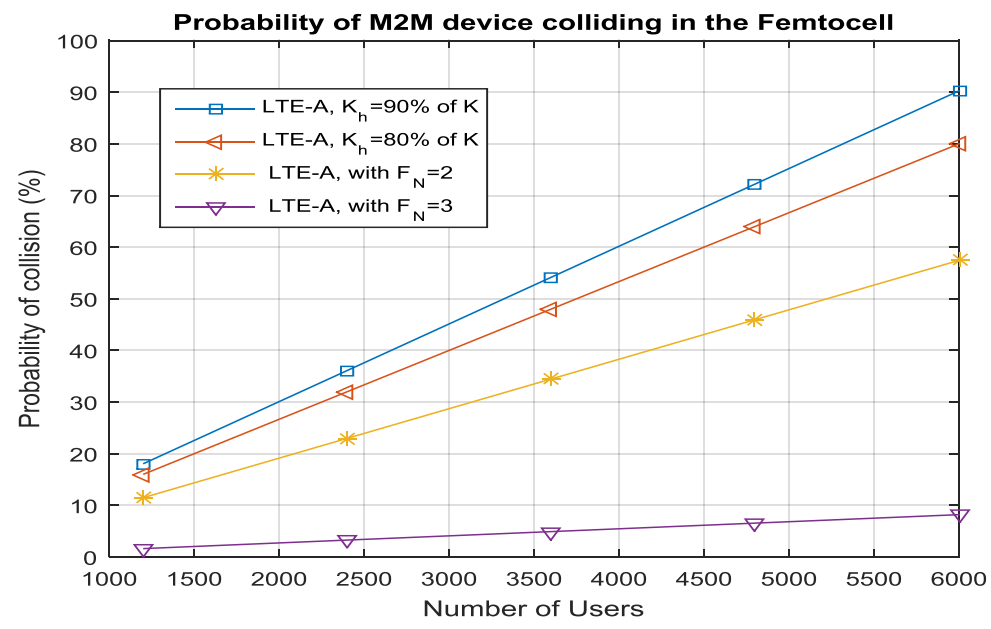

Figure 5. Probability of collision in RACH via femtocell

\section{CONCLUSION}

From the ensuing analysis it can be concluded that when an area is heavily populated with M2M devices and all these devices attempt to access the cellular network simultaneously, thus congestion is created on the random access channel which adversely affects the performance of the network not only for the M2M devices but also $\mathrm{H} 2 \mathrm{H}$ users. By employing Complete Frequency Reuse scheme one can assign resources independently to the femtocells which enable the M2M devices to gain access to the random access network through the femtocell.The adverse effect of many M2M devices attempting to access the network simultaneously can be mitigated by injecting more femtocells into the network to improve the possibility of a device gaining access to the network as well as improving the system's capacity and thereby avoiding the probability of a device colliding with other devices on the random access channel. This method overcomes the problem of the convention Fractional Frequency Reuse scheme in which the system's capacity decreases when the channel resources are shared among the contending sections. Again this method inproves the system's capacity by a factor of $(\mathrm{N}+1)$ and is devoid of computational complexity associated with the Two Level Power Control (TLPC) scheme. Here since the Femtocells are deployed by the network operator it is easy to implement.

\section{REFERENCES}

[1] A. Ghosh, R. Ratasuk, B. Mondal, Nitin Mangalvedhe and T. Thomas, "LTE-advanced: Next Generation Wireless Broadband Technology," IEEE Wireless Communications, Vol. 17, No. 3, pp. 10-22, June 2010.

[2] A. Khandekar, N. Bhushan, J. Tingfang and V. Vanghi, "LTE-advanced: Heterogeneous Networks," 2010 European Wireless Conference, pp. 978-982, 2010.

[3] D. Kai, L. Zhanjun, Z. Shiyan, R. Weiyi and L. Wei, "Research on Resource Allocation Based on Clustering Femtocell Networks," Indonesian Journal of Electrical Engineering and Computer Science (IJEECS), Vol. 7, No. 1, July 2017, pp. 138-149, DOI: 10.11591/ijeecs.v7.i1.pp138-149.

[4] K. Odeyemi and E. Ogunti, "Capacity Enhancement for High Data Rate Wireless Communication System," International Journal of Electrical and Computer Engineering (IJECE), Vol. 4, No. 5, October 2014, pp. 800-809, ISSN: 2088-8708.

[5] Cisco White Paper, "Cisco visual Network Index: Global Mobile Data Traffic Forecast Update", 2015-202", February 3, 2016

[6] O. Hayoung and L. Sangsoon, "Light-weight routing protocol in IoT-based inter-device telecommunication wireless environment", International Journal of Electrical and Computer Engineering (IJECE), Vol. 6 No. 5, pp. 23552-2361, ISSN: 2088-8708, October 2016. DOI: 10.11591/ijece.v6i5.10504.

[7] S. Deswal and A. Sighrova, "A Vertical Handover Algorithm in Integrated Macrocell-Femtocell Networks", International Journal of Electrical and Computer Engineering (IJECE), Vol. 7, No. 1, pp. 299-308, ISSN: 20888708, DOI: 1011591/ijece.v7i1, pp. 299-308, February 2017.

[8] R. Ghaffer and R. Knopp, "Fractional Frequency Reuse and Interference Suppression of OFDMA Networks," $8^{\text {th }}$ International Symposium on Modeling and Optimization in Mobile Ad Hoc, and Wireless Networks, Avignon, France, 2010, pp. 273-277

[9] "3G Evolution HSPA and LTE for Mobile Broadband"

[10] 3GPP R1-050629, “Inter-cell Interference Mitigation,” Huawei, 2005. Available (July 2012): http://www.3gpp.org/ftp/tsg ran/wg1 rl1/TSGR1 AH/LTEAH June-05/Docs/R1-050629.zip 
[11] 3GPP, Radio access network work group 1 contributions, http://www.3gpp.org, September 2005.

[12] I. Leyva-Mayorga, L. Tello-Oquendo, V. Pla. J. Martinez-Bauset and V. Cassres-Giner, "Performance Analysis of Access Class Barring for Handling Massive M2M Traffic in LTE-A Networks," 2016 IEEE International Conference on Communications (ICC), Kuala Lumpur, pp. 1-6, 2016.

[13] J. Park and Y. Lim, "Adaptive Access Class Barring Method for machine Generated Communication," Mobile Information Systems, Vol. 2016 (2016), ID 6923542, 6 pages, 2016. http://dx.doi.org/10.1155/2016/6923542

[14] S. S. Han, J. Park, T. Lee, H. G. Ahn and K. Jang, "A New Frequency Partitioning and Allocation of Subcarriers for Fractional Frequency Reuse in Mobile Communication Systems," IEICE Transactions on Communications, Vol. E91, Issue No. 8, pp 2748-2751, 2008.

[15] R. Giuliano, C. Monti and P. Loreti, "Wimax Fractional Frequency Reuse for Rural Environments," IEEE Wireless Communications, Vol. 15, Issue No. 3, pp60-65, 2008. DOI:10.1109/MWC.2008.4547524

[16] P. Teemu, L. Panu and L. Jukka, "Deployment of Mobile Wimax in Urban Environment," $11^{\text {th }}$ International Symposium on Wireless Personal Multimedia Communications (WPMC), 2008.

[17] I. N. Stiakogiannakis and D. I. Kaklamani, "Factional Frequency Reuse Techniques for Multi-Cellular Wimax Networks," $21^{\text {st }}$ Annual IEEE International Symposium on Personal, Indoor and Mobile Communications, 2010, pp. 2432-2437, DOI: 10.1109/PIMRC.2010.5671728

[18] A. Daeinabi, K. Sandrasegaran and X. Zhu, "An Intercell Interference Coordination Scheme in LTE Downlink Based on User Priority and Fuzzy logic System," International Journal of Wireless and Mobile Networks (IJMMN), Vol. 5, Issue No.4, pp. 1-16, August, 2013.

[19] V. Rekha, "Inter-Cell Interference Mitigation in Long Term Evolution Networks: A Survey," India Journal of Science and Technology, Vol. 9(48), DOI: 10.17485/ijst/2016/v9i48/107995, ISSN: 0974-5645

[20] N. Saquib, E. Hossain and D. I. Kim, "Fractional Frequency Reuse for Interference Management in LTE-Advanced HetNets," in IEEE Wireless Communications, Vol. 20, No. 2, PP. 113-122, April 2013, DOI; 10.1109/MWC.2013.6507402.

[21] M. T. Kawser, M. R. Islam, K. I. Ahmed, M. R. Karim and J. B. Saif, "Efficient Resource Allocation and Sectorization for Fractional Frequency Reuse (FFR) in LTE Femtocell Systems," Applications of Wireless Communications, Radio Engineering, Vol. 24, No. 4, 2015, DOI: 10.13164/re.2015.0940

[22] T. M. Lin, C. H. Lee, J. P. Cheng and W. T. Chen, "PRADA: Prioritized Random Access with Dynamic Access Barring for MTC in 3 GPP LTE-A Networks," in IEEE transactions on vehicular technology, Vol. 63, No. 5, pp. 2467-2472, DOI:10.1109/TVT.2013.2290128

[23] 3 GPP ETSI TS 136211 V10.4.0(2012-01), LTE: Evolved Universal Terrestrial Radio Access (E-UTRA); Physical Channels and Modulation (3GPPTS 36.211 version 10.4.9 Release 10) pp. 47

[24] K.S. Ko, M. J. Kim, K. Y. Bae, D. K. Sung, J. H. Kim and J. Y. Ahn, "A Novel Random Access for Fixed Location Machine to Machine Communications in OFDMA Based Systems," in IEEE Communications Letters, Vol. 16, No. 9, pp. 1428-1431, September 2012.

\section{BIOGRAPHIES OF AUTHORS}

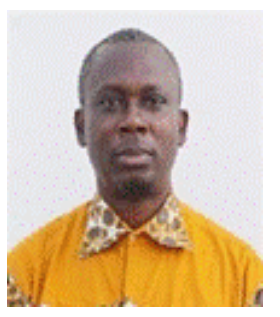

Eric Sackey received his M.Tech degree from the department of Electronic and Communications Engineering, SRM University, Channai, India. He received his Bachelor's degree in Electronics and Communications Engineering from All Nations University College, Koforidua, Ghana. His research interests include Wireless Communications, MIMO OFDM, machine to machine communications, LTE and LTE-Advanced Communication Systems.

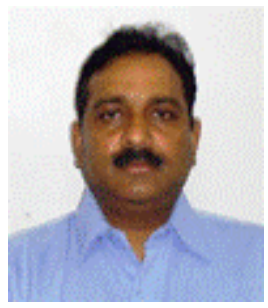

Dr. Rajeev Paulus received his Doctorate degree in Electronic \& Communication Engineering from SHUATS, Allahabad and M.Tech degree from the Department of Electrical Engineering, MNNIT, Allahabad. He received his Bachelor's degree in Electronic engineering from University of Pune. He has been working in the Department of Electronic \& Communication Engineering, as an Assistant Professor in Sam Higginbotom University of Agriculture, Technology \& Sciences. He has published number of Research paper in national, international journals and Conferences. His specializations include wireless communication and networks. His current research interests are Data Communication Networking, Optical communication \& network, Network Management, 4G, Sensor \& Adhoc-network. He is a member of the IEEE and a life member of ISTE. He is a member of advisory board in many National and International journal. 